\title{
Highly Sensitive 56 Gbps NRZ O-band BiCMOS-Silicon Photonics Receiver using a Ge/Si Avalanche Photodiode
}

\author{
S. A. Srinivasan ${ }^{1 *}$, J. Lambrecht ${ }^{2 *}$, M. Berciano ${ }^{1}$, S. Lardenois ${ }^{1}$, P. Absil ${ }^{1}$, J. Bauwelinck ${ }^{2}$, X. Yin ${ }^{2}$, M. \\ Pantouvaki $^{1}$, J. Van Campenhout ${ }^{1}$ \\ ${ }^{1}$ imec, Kapeldreef 75, 3001 Leuven, Belgium \\ ${ }^{2}$ IDLab, Department of Information Technology, Ghent University-imec, 9052 Ghent, Belgium \\ *These authors contributed equally \\ 'Authore-mail address: ashwyn.srinivasan@imec.be
}

\begin{abstract}
A hybrid BiCMOS-Silicon Photonics receiver with a waveguide-coupled $\mathrm{Ge} / \mathrm{Si}$ avalanche photodiode is demonstrated with OMA sensitivities of $-14.4 \mathrm{dBm}$ for error-free operation at $50 \mathrm{Gbps}$ and $-18.6 \mathrm{dBm}$ under the KP4-FEC limit at $56 \mathrm{Gbps}$ NRZ-OOK.

OCIS codes: (040.1345) Avalanche photodiodes, (130.3120) Integrated optics devices, (060.4510) Optical Communication
\end{abstract}

\section{Introduction}

With $400 \mathrm{~Gb} / \mathrm{s}$ optical transceivers starting to emerge in today's cloud datacenters, the development of nextgeneration transceivers operating at $800 \mathrm{~Gb} / \mathrm{s}$ and $1.6 \mathrm{~Tb} / \mathrm{s}$ is becoming imminent [1]. For such high-data-rate optical links, improving link margins is of key importance, either to increase the reach of the link or to reduce the number of required lasers and their associated cost and power consumption. To achieve this, avalanche photodiodes (APDs) have shown potential to enable higher receiver sensitivities and improved optical link margins operating at 50 Gbps and beyond data rates [2]. In this paper, a receiver comprising a silicon photonics waveguide-coupled Ge/Si APD, wirebonded to a SiGe BiCMOS transimpedance amplifier (TIA) is presented. The receiver has an optical modulation amplitude (OMA) sensitivity of $-18.6 \mathrm{dBm}$ under the KP4-FEC limit at 56Gb/s NRZ-OOK, and -14.4 $\mathrm{dBm}$ running error-free at $50 \mathrm{~Gb} / \mathrm{s}$ NRZ-OOK. To the best of our knowledge, this is among the highest reported sensitivities for Ge/Si APD-based receivers, while consuming just $140 \mathrm{~mW}$.

\section{Device fabrication and performance}

The waveguide coupled Ge/Si APDs presented in this work were fabricated using imec's $200 \mathrm{~mm}$ Silicon Photonics Platform along with other passive and active waveguide components [2-3]. The devices use a lateral separateabsorption-charge-multiplication (SACM) implementation [2], with an epitaxial Ge waveguide acting as the absorption layer, and the charge and multiplication regions implemented in the $220 \mathrm{~nm}$ Si device layer. A crosssectional schematic of the device can be found in the inset of Fig 1 . The doping profiles for the charge region in the device were optimized to have high electric fields in the multiplication region, while maintaining sufficient electric field in the absorption region to extract the photo-generated carriers. The device is coupled to a $220 \mathrm{~nm}$ thick Si waveguide using low-loss poly-Si tapers, and a grating coupler for TE polarization with peak coupling at $1310 \mathrm{~nm}$ was used for fiber-to-chip optical coupling. Fig. 1 shows the wafer-scale statistics of the APD's responsivity and dark current when illuminated at $1310 \mathrm{~nm}$ with an input optical power of $-20 \mathrm{dBm}$. The operating bias voltage range is limited to $-13 \mathrm{~V}$, beyond which the device fails catastrophically [4]. The small-signal characteristics of the device were also measured, and the extracted $3 \mathrm{~dB}$ opto-electrical bandwidth $\left(\mathrm{f}_{3 \mathrm{~dB}}\right)$ are shown in Fig. 2. From the increase in measured RF power at increasing operating bias, we extract a multiplication gain (M) of 10 . The resulting gainbandwidth-product (GBP) of the device is $300 \mathrm{GHz}$ at $-13 \mathrm{~V}$.
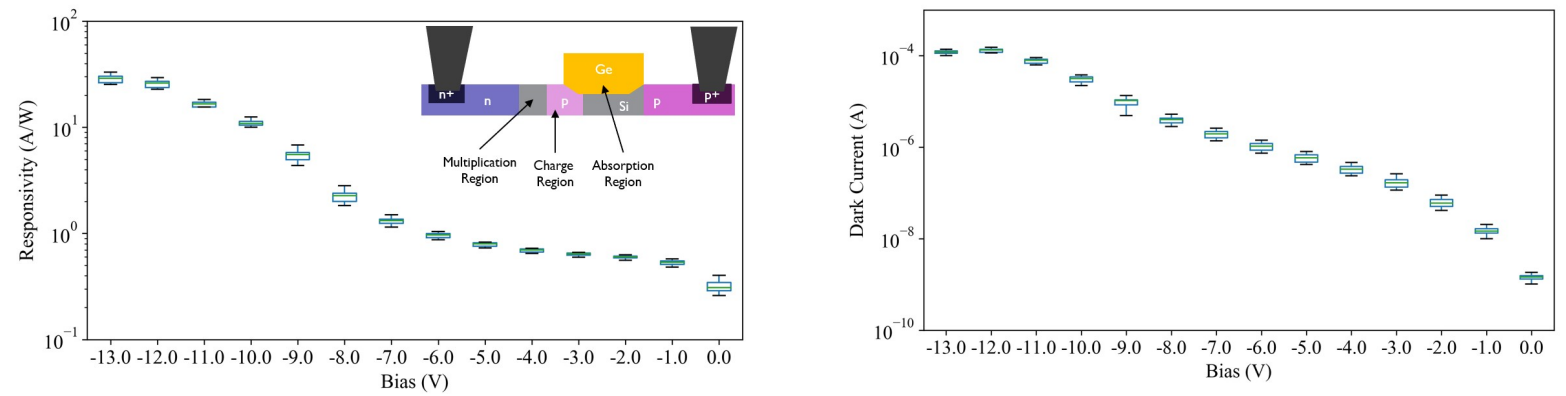

Fig.1 Wafer-scale responsivity and dark current measured from Ge/Si APD across 30 dies in a $200 \mathrm{~mm}$ wafer, with a cross-sectional schematic of the device in the inset [2]. The measurement was performed at $1310 \mathrm{~nm}$ and at an optical power of $-20 \mathrm{dBm}$. 


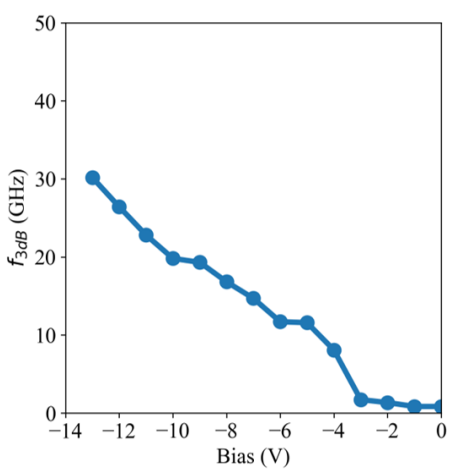

(a)

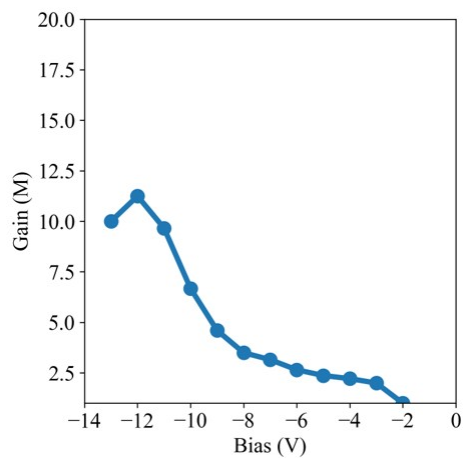

(b)

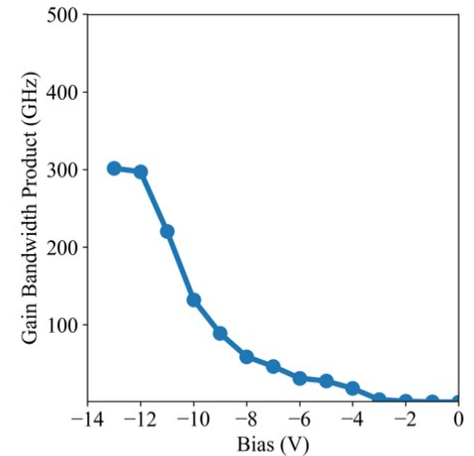

(c)

from small-signal measurements.

\section{Experiment setup and results}

The TIA chip in the receiver presented in this work was fabricated in a $55 \mathrm{~nm}$ SiGe BiCMOS technology. Nominally, it consumes $140 \mathrm{~mW}$ for $50-56 \mathrm{~Gb} / \mathrm{s}$ operation. However, at the cost of maximum $2 \mathrm{~dB}$ sensitivity penalty, its power consumption can be reduced further down to $1.8-2 \mathrm{pJ} / \mathrm{bit}(100 \mathrm{~mW})$ [4]. The TIA chip and the APD were wirebonded to a test PCB as seen in Fig. 3 (a). The APD supply voltage was set to -10.5 V, which resulted in $-10 \mathrm{~V}$ effective reverse bias over the device. Fig. 3 (b) shows the experimental setup used for the biterror-rate measurements. A $92 \mathrm{GSa} / \mathrm{s}$ arbitrary waveform generator (AWG) was used to generated $50 \mathrm{~Gb} / \mathrm{s}$ and 56 $\mathrm{Gb} / \mathrm{s} 2^{15}-1$ pseudorandom binary (PRBS) input signals in NRZ-OOK modulation format. A $1310 \mathrm{~nm}$ laser was modulated by a $35 \mathrm{GHz}$ Mach-Zehnder Modulator driven by a $50 \mathrm{GHz}$ RF amplifier. The optical eye sent to the silicon photonics receiver was measured using a $70 \mathrm{GHz}$ DC-coupled reference photodetector. The measured extinction ratio was $7.5 \mathrm{~dB}$ for all data rates. The BER was measured by a SHF 11100B error analyzer (EA).
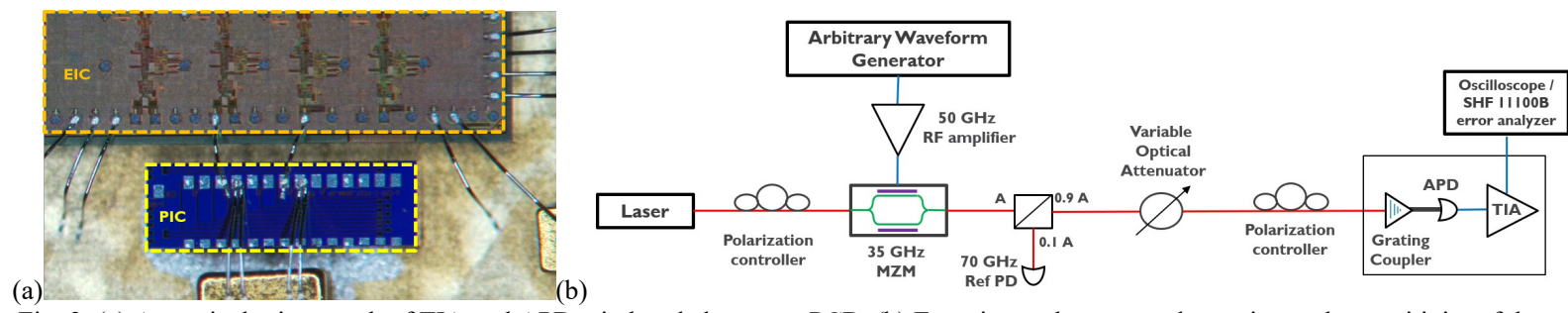

Fig. 3. (a) An optical micrograph of TIA and APD wirebonded to a test PCB. (b) Experimental setup used to estimate the sensitivity of the receiver.

Fig. 4 shows the measured transmitted optical eye from the MZM and the eye from the receiver with APD through the TIA. The BERs plotted vs the received OMA are shown in Fig. 5. The OMA is referred to the input waveguide of the APD using the measured average photocurrent at $-3 \mathrm{~V}$ where the responsivity is accurately measured. Fig. 5 shows that the receiver has the potential to operate error-free $\left(B E R<10^{-12}\right)$ with an OMA sensitivity of $-14.4 \mathrm{dBm}$ and $-11.6 \mathrm{dBm}$ for $50 \mathrm{Gbps}$ and $56 \mathrm{Gbps}$ NRZ-OOK modulation respectively. Compared to a standard Ge/Si p-i-n photodiode, wirebonded to the same TIA as reported in [4], the APD receiver shows a sensitivity improvement of $\sim 1.8 \mathrm{~dB}$ for $\mathrm{BER}=10^{-12}$ at $50 \mathrm{Gbps}$ data rates, while matching the same OMA sensitivity at $56 \mathrm{Gbps}$. Under the KP4FEC limit, the APD receiver has a sensitivity improvement of $4.4 \mathrm{~dB}$ and $3 \mathrm{~dB}$ for $50 \mathrm{Gbps}$ and $56 \mathrm{Gbps}$ respectively compared to the $\mathrm{p}$-i-n photodiode.
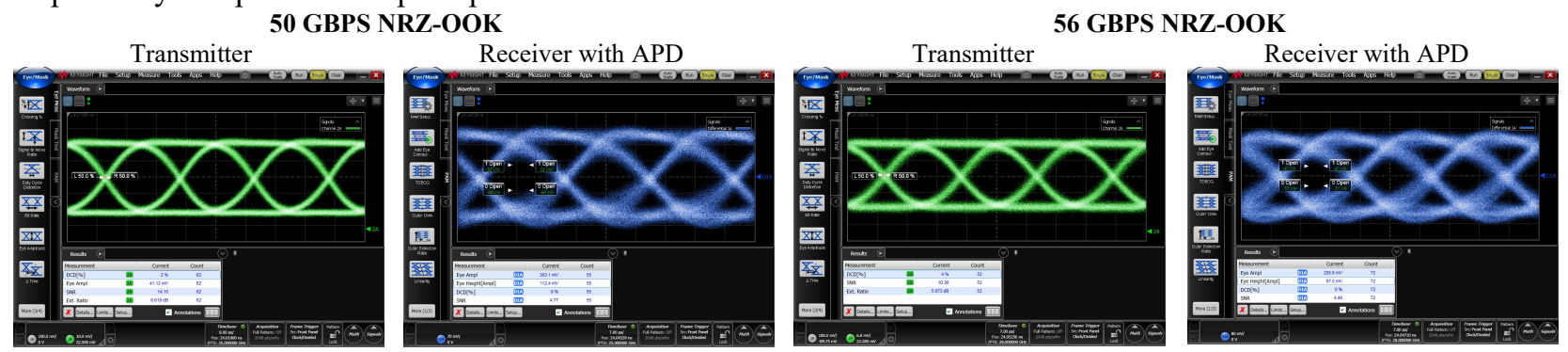

Fig 4. Optical and electrical eyes of the transmitter and receiver with the Ge/Si APD at 50 Gbps and 56 Gbps NRZ-OOK data rates. 


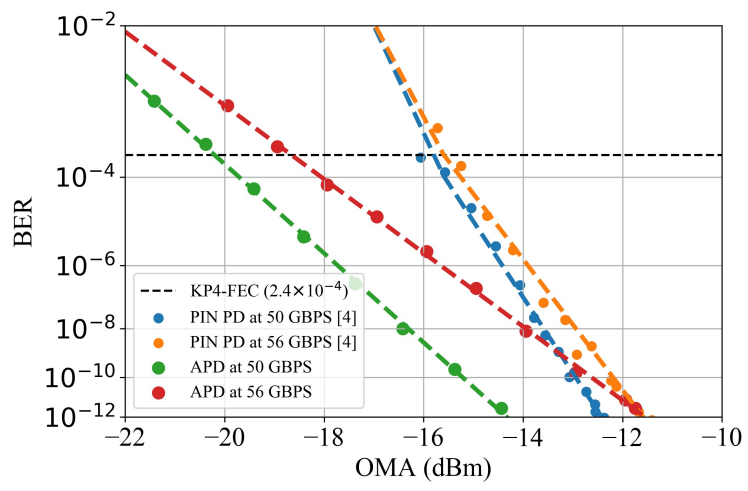

Fig. 5. BER measured with an error detector. The APD was biased at $-10 \mathrm{~V}$ and measured at 1310 nm, whereas the p-i-n PD in [4], was measured at $1550 \mathrm{~nm}$.

It should be noted that receivers with standard $\mathrm{Ge} / \mathrm{Si} \mathrm{p}$-i-n photodiode and reported in [4] were measured in the Cband, whereas the APDs in this work were measured at O-band. In Table I, we compare our BER results with current state-of-the-art Ge/Si based APDs. On comparing with other published results, our receiver achieves some of the best sensitivities for KP4-FEC BER limits.

Table I: Benchmarking with selected prior published Ge $/ \mathrm{Si}$ APD-based receivers at $1310 \mathrm{~nm}$.

\begin{tabular}{|c|c|c|c|c|c|c|c|}
\hline Ref & Device type & Data rate & Voltage (V) & Dark Current (A) & Gain (M) & BW (GHz) & OMA sensitivity \\
\hline$[5]$ & Fiber coupled & $53.125 \mathrm{~Gb} / \mathrm{s}$ & -11.38 & $3 \times 10^{-6}$ & 7 & 20 & $-16 \mathrm{dBm}, \mathrm{KP} 4-\mathrm{FEC}$ \\
\hline$[6]$ & Waveguide coupled & $56 \mathrm{~Gb} / \mathrm{s}$ & -3.95 & $\mathrm{NA}$ & $\mathrm{NA}$ & $>20$ & $-12 \mathrm{dBm}, \mathrm{BER}=2 \times 10^{-5}$ \\
\hline This & Waveguide & $56 \mathrm{~Gb} / \mathrm{s}$ & -10 & $3 \times 10^{-5}$ & 10 & 20 & $-18.6 \mathrm{dBm}, \mathrm{KP} 4-\mathrm{FEC}$ \\
work & coupled & $50 \mathrm{~Gb} / \mathrm{s}$ & -10 & $3 \times 10^{-5}$ & 10 & 20 & $-14.4 \mathrm{dBm}, \mathrm{BER}=1 \times 10^{-12}$ \\
\hline
\end{tabular}

\section{Conclusion}

A silicon photonics receiver consisting of a waveguide integrated $\mathrm{Ge} / \mathrm{Si}$ avalanche photodiode wirebonded to TIA fabricated in $55 \mathrm{~nm} \mathrm{SiGe} \mathrm{BiCMOS}$ technology is presented. The receiver operates at O-band and has an OMA sensitivity of $-18.6 \mathrm{dBm}$ under the KP4-FEC limit at $56 \mathrm{~Gb} / \mathrm{s}$ and $-14.4 \mathrm{dBm}$ running error-free at $50 \mathrm{~Gb} / \mathrm{s} \mathrm{NRZ-}$ OOK. The APD enables a sensitivity improvement of 1.8 to $4.4 \mathrm{~dB}$ when compared to a standard $\mathrm{Ge} / \mathrm{Si}$ p-i-n photodiode. A receiver with such a device has the potential in improving optical link margins for $400 \mathrm{GbE}$ and beyond applications.

\section{Acknowledgements}

The Ge/Si avalanche photodiode was developed as part of imec's industrial affiliation R\&D program on Optical I/O. The work of J. Lambrecht was supported by the Research Foundation Flanders (FWO). This work was supported partly by EU H2020 Projects PICTURE (Grant 780930) and TERABOARD (Grant 688510).

\section{References}

[1] 2018 Ethernet Alliance Roadmap - www.ethernetalliance.com

[2] S.A. Srinivasan, et al. "Silicon-contacted waveguide integrated Ge/Si avalanche photodiode with $32 \mathrm{GHz}$ bandwidth and multiplication gain >8." European Conference on Optical Communication (ECOC), 2019.

[3] M. Pantouvaki, et al. "Active components for $50 \mathrm{~Gb} / \mathrm{s}$ NRZ-OOK optical interconnects in a silicon photonics platform." Journal of Lightwave Technology 35.4: 631-638, 2017.

[4] J. Lambrecht, et al. "90-Gb/s NRZ Optical Receiver in Silicon Using a Fully Differential Transimpedance Amplifier." Journal of Lightwave Technology 37.9: 1964-1973. 2019.

[5] S. Park, et al. "50-Gbps Receiver Subsystem using Ge/Si Avalanche Photodiode and Integrated Bypass Capacitor." 2019 Optical Fiber Communications Conference and Exhibition (OFC). IEEE, 2019.

[6] A. Samani, et al. "Highly Sensitive, 112 Gb/s O-band Waveguide Coupled Silicon-Germanium Avalanche Photodetectors." Optical Fiber Communication Conference. Optical Society of America, 2019. 